\title{
O Rato Sniffy Vai à Escola ${ }^{1}$
}

\author{
Gerson Yukio Tomanari² \\ Universidade de São Paulo \\ David Alan Eckerman \\ University of North Carolina at Chapel Hill
}

\begin{abstract}
RESUMO - Sniffy Pro - O Rato Virtual é um programa de computador, acompanhado de um manual de laboratório, que tem como proposta servir de recurso didático aplicado ao ensino introdutório de Análise Experimental do Comportamento, em especial às atividades práticas normalmente desenvolvidas em laboratórios de condicionamento operante que empregam ratos como sujeitos e caixas de Skinner como equipamento experimental. O objetivo do presente artigo é analisar Sniffy Pro no tocante aos aspectos concernentes à sua pertinência e adequação como material de ensino. Segundo os autores de Sniffy Pro, este aplicativo seria atraente pelo que representa em termos de tecnologia informatizada, pelo seu baixo custo, pela sua facilidade de uso e pela economia de tempo que propicia. Entretanto, tomados vários aspectos da concepção de Sniffy Pro, seu conteúdo e estrutura, bem como alguns aspectos éticos relacionados, demonstra-se que a sua utilização como recurso didático deve muito provavelmente ocorrer às custas de prejuízos na formação científica do estudante de Psicologia.
\end{abstract}

Palavras-chave: Sniffy Pro; atividades de laboratório; material de ensino.

\section{Sniffy The Rat Goes to School}

\begin{abstract}
Sniffy Pro - The Virtual Rat is a computer software program as well as a laboratory textbook developed to serve as an alternative teaching tool to be used in introductory courses on the Experimental Analysis of Behavior, especially the laboratory activities that usually employ rats as experimental subjects and Skinner boxes as apparatuses. The main goal of this paper was to analyze Sniffy Pro regarding its adequacy as a learning resource. According to the authors of Sniffy Pro, this software would be attractive because it applies technology to a teaching setting, by its low cost, by its ease of use and by the time it saves. However, the present analysis of Sniffy Pro's conception, content, structure, as well as ethical issues, allows one to conclude that its use as a teaching tool is likely to impair the student's learning.
\end{abstract}

Key words: Sniffy Pro; laboratory activities; learnig resource.

\begin{abstract}
Quando Pedro Preguiça entrou na escola com seus amigos, viu Dona Quati, a professora, caminhando em sua direção. Ela falava com todas as crianças e, quando viu Pedro, tomoulhe as patinhas nas suas patas, pedindo-lhe que lhe dissesse seu nome.

- Meu nome é Pedro, respondeu ele. Eu estava doente. Estava com sarampo. É por isso que eu não vim antes para a escola. - Não tem importância, disse Dona Quati. Você não perdeu nada que ainda não possa recuperar.
\end{abstract}

(“Pedro Preguiça vai à escola”, F. S. Keller, 1987)

A Análise Experimental do Comportamento (AEC) constitui parte predominante de muitos currículos de graduação em Psicologia no Brasil, muito freqüentemente representando a Psicologia Experimental. Um currículo típico de AEC inclui tanto os seus aspectos filosóficos (Behaviorismo) quanto os

1 A realização deste trabalho contou com a ajuda financeira do $\mathrm{CNPq}$ por meio da Bolsa Produtividade em Pesquisa concedida ao primeiro autor. Os autores agradecem os valiosos comentários da Profa. Maria Amelia Matos, bem como à Sociedade Brasileira de Psicologia (SBP), que acolheu a conferência "O Rato Sniffy vai à escola" proferida pelos autores na ocasião da XXXII Reunião Anual realizada na Universidade Federal de Santa Catarina, Florianópolis, em outubro de 2002.

2 Endereco: IPUSP - Av. Prof. Mello Moraes, 1721, São Paulo, SP 05508-900. Email: tomanari@usp.br seus conceitos fundamentais - demonstrados, estes últimos, no laboratório de condicionamento operante, geralmente utilizando-se ratos como sujeitos e caixas de Skinner como equipamento experimental (Gomide e Weber, 1998; Guide e Bauermeister, 1979; Matos e Tomanari, 2002).

O laboratório de AEC constitui, certamente, uma das raras oportunidades de os alunos contatarem a Psicologia na perspectiva de uma ciência experimental, na qual estes podem observar e analisar o comportamento sob função de fatores ambientais - conseqüientes e antecedentes - com os quais um organismo vivo interage. Fundamentalmente, no nível de graduação, o laboratório de AEC tem como objetivo propiciar condições para que os alunos observem, identifiquem e demonstrem princípios comportamentais. Em se tratando de processos básicos, em geral são tratados tópicos como reforçamento, extinção, esquemas simples e complexos de reforço, encadeamento de respostas, discriminação e generalização de estímulos etc. Quando se incluem fenômenos complexos no ser humano, os alunos em geral estudam, no laboratório, comportamento verbal, aprendizagem relacional, formação de conceitos, processos de simbolização por equivalência de estímulos etc.

O laboratório de AEC desenvolve, por meio de atividades essencialmente práticas, habilidades específicas ligadas aos princípios conceituais e metodológicos da área, tais como, por exemplo, observar sistematicamente um organismo vivo que 
se comporta no ambiente proposital e relativamente simples da caixa de Skinner; identificar e estabelecer relações funcionais entre o comportamento do organismo e o ambiente com o qual interage; manipular controladamente variáveis ambientais que atuam como eventos antecedentes e conseqüentes a um dado comportamento; acompanhar os efeitos de tais manipulações sobre o comportamento do organismo por meio de registros e análises de dados sistemáticos.

Em um certo sentido, habilidades como essas que envolvem a capacidade de analisar comportamentos constituem o repertório de formação nuclear do psicólogo e, por isso, transcendem os limites do contexto do laboratório. Sob as condições controladas e prototípicas do laboratório, os alunos aprendem a observar, medir, registrar, enfim, analisar o comportamento de um organismo vivo na situação privilegiada de interação em que os comportamentos do sujeito e do aluno-investigador modificam-se mutuamente. Trata-se, sem dúvida, de uma unidade celular, um modelo reduzido de interação, na qual a capacidade de os alunos de analisar comportamentos encontra condições propícias para ser adquirida em suas bases fundamentais.

A título de exemplo, o laboratório de AEC expõe os alunos a diferentes métodos e delineamentos de pesquisa que lidam, de diferentes maneiras, com dados que refletem similaridades e, mais freqüentemente, diferenças individuais. Pode-se afirmar que, no laboratório de AEC, vigora a heterogeneidade, pois, em cada instância de análise, os alunos deparam-se com tantos diferentes padrões de comportamento quantos são os sujeitos experimentais. Em um certo sentido, esta diversidade reflete-se, marcadamente, nas explicações imediatas dadas pelos estudantes para o comportamento de seu rato quando os resultados obtidos ao fim de um exercício não são exatamente aqueles que eles esperam: “o estúpido não aprende nada, ele está com sono, ele não estava com fome, ele estava doente, e assim por diante" (Keller, 1968, p. 88). O desafio, nesses casos, é trabalhar com os alunos o fato de que "o rato", como Keller dizia, "está sempre certo" (idem, p. 88) e que a compreensão do que se observa dar-se-á pela análise de fatores complexos, muitas vezes guardados na história particular de exposição de cada animal às suas próprias contingências e desvendados pelo delineamento experimental típico da área, o delineamento de 'sujeito como seu próprio controle' ou 'delineamento de sujeito único' (Sidman, 1960).

O delineamento de sujeito único tem como objeto de análise o comportamento de cada organismo, individualmente, respeitando-se, tanto as singularidades da sua história de vida, quanto aquelas relativas a cada diferente contexto que se apresenta; para fins da análise experimental, são promovidas alterações sistematicamente programadas nos contextos ambientais a que os sujeitos são expostos de modo que os efeitos de variáveis isoladas sobre o comportamento podem ser estudados. Na medida em que cada organismo vivo constitui um ser único, e que o contexto ambiental é marcado por seu moto contínuo de alterações, não seria este delineamento a essência dos estudos psicológicos (estudos de casos, por exemplo), muito mais do que a comparação entre diferentes sujeitos ou grupos de sujeitos, submetidos a diferentes condições experimentais e comparados por recursos estatísticos?
A depender de como o professor propõe as atividades de laboratório, estas podem vir a fomentar, nos estudantes, um conjunto de atitudes científicas perante os mais diversos fenômenos psicológicos que lhes possam apresentar. Neste caso, inserem-se as atividades do laboratório no contexto em que os alunos não se colocam apenas como consumidores, mas como produtores de conhecimento, aprendizes de métodos e técnicas que os qualificam para tal (Machado e Matos, 1990; Tomanari, 2000).

No conjunto das atitudes científicas, o laboratório fornece condições para se evidenciar a importância dos registros sistemáticos de dados. Estes registros destacam fenômenos comportamentais muitas vezes inacessíveis aos olhos da observação não sistemática, e permitem, ao aprendiz, proceder com diferentes tratamentos e análises de dados, fornecendo-lhe condições de formular uma base empírica de compreensão do fenômeno comportamental que estuda. Por não lidar com explicações finais, irrefutáveis, ou essencialmente teóricas, dá-se no laboratório a possibilidade de que os alunos vejam como um conjunto de idéias predominantes inter-relaciona-se com dados recém-produzidos e integrem-se na dinâmica do produzir conhecimento científico.

No laboratório, os alunos aprendem a lidar com os sucessos e os fracassos de uma predição. Esses últimos, a propósito, acabam por constituir um contexto propício aos alunos confrontarem os dados por eles coletados com aqueles disponíveis na literatura, sob o qual podem resultar propostas de investigações que propiciarão o avanço do conhecimento - para isso, o professor tem um papel fundamental. Esta é uma oportunidade na qual o laboratório traz para o contexto didático a possibilidade de os alunos se depararem com um dos aspectos marcantes da rotina do trabalho científico, isto é, o fato de que os resultados de uma pesquisa levam o pesquisador a uma série de questionamentos subseqüentes. É também a oportunidade na qual o professor pode cultivar em seus alunos o espírito crítico e investigativo, na tentativa de distanciá-los de dados irrefutáveis, hipóteses impossíveis de serem testadas, resultados oriundos de pesquisas com erros metodológicos etc.

\section{Sniffy Pro: Um recurso didático?}

Adicionalmente aos recursos de um laboratório animal que propiciam as oportunidades didáticas acima apontadas, os professores de AEC contam há algum tempo com uma ferramenta didática alternativa, o "Sniffy Pro - The Virtual Rat" (Alloway, Wilson, Graham e Krames, 2000). Trata-se de um programa de computador desenvolvido por Greg Wilson, sob a orientação de três psicólogos (Tom Alloway, Jeff Graham e Lester Kramer) e consultores (Douglas Chute, entre eles). Este programa acompanha um manual de laboratório que instrui o aluno a realizar uma série de exercícios tendo como base um curso de laboratório que se destinaria a alunos de graduação trabalhando com um rato de experimentação.

O programa Sniffy $\mathrm{Pro}^{3}$ exibe um rato em uma caixa de

3 Empregamos "Sniffy Pro" para designar o conjunto de partes que integram este instrumento de ensino, isto é, o programa de computador e o manual de laboratório. Quando nos referimos ao rato virtual, em si, empregamos simplesmente "Sniffy". 
Skinner e inclui 40 seqüências de movimentos adaptadas a partir de 600 quadros extraídos do vídeo de um rato movimentando-se no interior de uma caixa de Skinner.

Parte dos objetivos do programa é motivada por princípios altamente louváveis. Os autores temem que os alunos de Psicologia, caso não tenham a oportunidade de acesso a um laboratório real, fiquem alheios aos procedimentos e experiências que um trabalho em laboratório propicia e, igualmente, aos fenômenos comportamentais básicos. Os autores preocupam-se em fazer com que os alunos aprendam como os experimentos são montados, como os dados são coletados e analisados e como se chega a conclusões que tenham dados como base.

Os autores enfatizam, ainda, o quanto Sniffy difere de um rato real e em que medida o trabalho com Sniffy Pro não substitui o trabalho com um rato real, posto que constitui tão somente uma metáfora. Eles enfatizam que Sniffy Pro pode apenas mostrar aquilo que os psicólogos propõem como verdadeiro e que o trabalho com Sniffy não tem o caráter de pesquisa; para continuar a explorar o comportamento de ratos, seria necessário trabalhar com animais reais. Os autores chegam até a mencionar dois dos vários aspectos em que Sniffy difere de um rato real: (1) ele não demonstra saciação, o que o faz estar sempre pronto a comer; (2) ele aprende mais rapidamente do que um rato real. Estas alterações são mostradas como positivas, uma vez que, segundo os autores de Sniffy, (1) raramente a motivação está entre os temas abordados em um curso sobre aprendizagem, tornando-a, assim, um fator de distração e (2) uma acelerada aprendizagem permite o estudo de um número maior de tópicos curriculares durante o período letivo.

Os autores prosseguem enfatizando que Sniffy oferece uma característica nova, a qual não é encontrada em um rato real - a "Janela da Mente" (Mind Window) - que mostra os princípios elementares que forneceriam a base dos fenômenos comportamentais demonstrados nos exercícios feitos em laboratório. A aprendizagem da resposta de pressionar a barra é representada na Janela da Mente por duas associações, Som-Comida e Barra-Som, representando, respectivamente, a formação das associações entre o som do funcionamento do mecanismo do comedouro e a apresentação de comida (associação som-comida presente no treino ao comedouro) e também a associação entre a resposta de pressionar a barra e o som do comedouro (associação barra-som presente no condicionamento da resposta de pressionar a barra). Esse relato não é aquele que muitos dos pesquisadores que trabalham com comportamento operante selecionariam, uma vez que a visão apresentada pelos autores é, talvez, mais compatível com aquela de Thorndike (Thorndike, 1898) do que com a de Skinner (Skinner, 1938). Dessa forma, contestamos a afirmação de que Sniffy Pro "simula e exibe alguns dos processos psicológicos em que os psicólogos acreditam (e que as pessoas) empregam" (p. 3). Nada contra as simulações computacionais em si (ver Kemp e Eckerman, 2001a, para uma discussão sobre os papéis de simulações), mas sim à Janela da Mente que, na realidade, é uma versão simplificada do modelo de aprendizagem de Rescorla-Wagner (e.g., 1972), ampliada para o comportamento operante (Daly e Daly, 1982, apresentam uma proposta nesse sentido; Kemp e Eckerman, 2001b, discutem algumas falhas dessa proposta). É preciso ter claro, entretanto, algo que os próprios autores de Sniffy parecem desconhecer. Isto é, o modelo de Rescorla-Wagner não tem sido preconizado por nenhum pesquisador na área como um modelo adequado de aprendizagem associativa e, menos ainda, de aprendizagem operante (Kemp e Eckerman, 2001b).

A Janela da Mente exige que explicitemos algumas possíveis implicações negativas de seu uso. À exceção de Sniffy, um processo de aprendizagem pode ser identificado pelos alunos ao observarem mudanças comportamentais funcionalmente relacionadas com a interação entre um organismo e o seu ambiente. Em se tratando do Sniffy, todavia, a Janela da Mente - e não o comportamento do organismo - identifica e mostra aos alunos se a aprendizagem ocorreu, em que momento e em que medida. Ou seja, trata-se do uso de um artefato, uma suposta variável interveniente que, além de irreal, reflete a negligência de Sniffy Pro com relação à importância de que os alunos passem pelo treino de observação sistemática do organismo vivo que se comporta, sobretudo em um laboratório didático, no qual, sob condições maximizadas de controle de variáveis, aprenderão a analisar os efeitos de diferentes contingências sobre o comportamento do organismo.

Mais ainda, ao fazerem uso da Janela da Mente para avaliar a aprendizagem de Sniffy, os alunos podem ser inadequadamente conduzidos a conceber a mente como a causa do comportamento. Isto seria muito negativo em termos de ensino de AEC, uma vez que essa concepção errônea afastaria os alunos da própria análise de contingências e dos reais fatores responsáveis pela manutenção e modificação do comportamento. Por exemplo, um aluno pode, de forma incorreta, embora plausível, assumir que Sniffy pressiona a barra porque o nível da associação Barra-Som é quantitativamente elevado, e não porque uma história particular de exposição a contingências tenha construído uma história de vida que, nas condições presentes, torna esse comportamento altamente provável. Nesse sentido, Sniffy Pro estabelece condições propícias para que os alunos tomem equivocadamente uma associação de eventos, nos aspectos que tangem ao procedimento em si, como uma pseudo-explicação de aprendizagem. De acordo com os autores, a Janela da Mente mostra "processos psicológicos" inferidos. Ao redigirem as rotinas de programação da Janela da Mente, os programadores de Sniffy tomaram como base a mente de um rato ou o seu comportamento observável, funcionalmente relacionado com modificações ocorridas no ambiente? Os autores respondem que a segunda alternativa é verdadeira, desde que os movimentos de Sniffy foram compilados de filmagens de ratos se comportando. Em suma, acreditamos que a "Janela da Mente" ensina aos alunos uma visão equivocada da Análise Experimental do Comportamento na medida em que os afasta da observação do organismo se comportando, do ambiente com o qual interage, e da relação funcional entre ambos.

O manual que integra Sniffy Pro faz uma breve análise explicativa sobre o condicionamento operante. Esse material, se somado no decorrer das seções do texto, totaliza 22 páginas $-13 \%$ do texto (contamos 172 páginas de conteúdo sobre AEC no manual). Os exercícios de condicionamento operante (1 a 19) cobrem a maior parte dos conteúdos que são ensinados na graduação, exceto com relação aos exercícios sobre Peak Shift (alteração de pico) e contraste comportamen- 
tal, que não constituem exercícios normalmente explorados nesse nível de formação. Por outro lado, notamos o fato de o tópico "encadeamento de respostas" não ter sido abordado no manual. Isto é uma pena, pois o encadeamento constitui um caminho inicial pelo qual os alunos entram em contato com o comportamento sob formas mais complexas. Devemos enfatizar, contudo, que se observa um grande esforço dos autores no sentido de descrever os procedimentos e processos de estabelecimento de um operante: determinação do nível operante, realização de treino ao comedouro e, em seguida, modelagem da resposta de pressão à barra. Para nós, este constitui o principal diferencial do texto. A exceção fica por conta de aspectos específicos. Acreditamos, na realidade que, em virtude da super simplificação do programa e da eliminação dos efeitos da saciação, os alunos que aprendem a modelar a resposta de pressão à barra utilizando Sniffy Pro poderão eventualmente demorar um pouco mais a aprender a modelar essa resposta na vida real. Este é um aspecto que pode ser testado e, talvez, até mesmo corrigido, aprimorando a simulação do computador. Entretanto, esta correção não eliminaria o fato de que a modelagem do Sniffy reduz-se a uma técnica que ignora o número infinito de modos pelos quais o comportamento pode ser gradualmente modificado (Machado \& Silva, 1998). Ao contrário, a modelagem do Sniffy ocorre - e sempre ocorrerá - dentro dos limites daquilo que está programado. Esta limitação permite-nos entender as razões pelas quais os autores fornecem as instruções explícitas sobre a sequiência mais eficaz para se modelar a resposta de pressão à barra: primeiro, deve-se treiná-lo a se levantar nas patas traseiras em algum ponto da caixa de Skinner; em seguida, a se levantar nas patas traseiras contra a parede do fundo e, por fim, a se levantar nas patas traseiras cada vez mais perto da barra. Em certo sentido, abordar a modelagem como um conjunto de regras confere a esta o restrito caráter de técnica; aplicada a um organismo vivo, muito provavelmente, vai revelar-se um conjunto de regras ineficientes, simplesmente porque há tantas formas de se modelar um comportamento quanto há variações e dimensões deste.

Dentro dos limites de uma técnica, entretanto, seria possível conceber que uma simulação de modelagem poderia, desde que bem feita, resultar em uma prática útil que acabaria aprimorando a habilidade dos alunos de modelar o comportamento de um rato. Concordamos que, no manual que integra Sniffy Pro, o fenômeno da modelagem é descrito de maneira interessante. Nesse sentido, é divertido ler a seção na página 31 e a seguinte, que estimula o aluno a aplicar esses princípios no treino de um gato. É bem possível, na verdade, que o autor dessa seção tenha um gato extremamente dócil. Em nossa experiência, a tarefa de treinar um gato exige toda a competência de um adestrador experiente, o que torna essa tarefa um território perigoso para um jovem estudante, uma vez que este poderá fracassar, não identificar as razões disso e, assim, comprometer o seu aprendizado.

Há ainda outro comprometimento relativo à modelagem que merece ser mencionado. Os autores observam, de forma correta, que a modelagem funciona apenas porque o comportamento é variável e, dessa forma, a simulação inclui um processo de randomização que parece resultar de variação aleatória. Há um modo importante em que a variação do comportamento não ocorre, de forma alguma, randomica- mente, e que a idéia mesmo de variação randômica acaba por violar. Acreditamos que o cérebro seja altamente complexo e suscetível a um sem número de fatores extrínsecos e intrínsecos. Não acreditamos, contudo, que o cérebro funcione de forma randômica. E, portanto, acreditamos que a variação seja altamente sistemática e que a caracterização da variação como randômica não faz mais do que empobrecê-la. A modelagem, na realidade, tira proveito da variação sistemática do comportamento - como é demonstrado pelo conhecido fenômeno da automodelagem da resposta de pombos de bicar um disco iluminado (Brown e Jenkins, 1968). A primeira bicada pode ser provocada por um estímulo associado com comida por meio de um processo sistemático, que nada tem de randômico. Um dos motivos da preocupação com relação à randomização do comportamento é que as flutuações que ocorrem no comportamento de Sniffy nunca farão uma boa simulação do comportamento real se a variação ocorrer ao sabor do acaso. Quem quer que já tenha modelado um rato privado a pressionar uma barra muito dificilmente deve ter visto o rato distanciar-se do comedouro logo após este ter obtido uma pelota de comida ou uma gota d'água. Não é o que ocorre com Sniffy.

O programa Sniffy Pro contempla fenômenos comportamentais já estudados experimentalmente em laboratório. Entre eles, há o fenômeno de Jorro de Respostas sob Extinção. Após estar sob reforçamento da resposta de pressionar a barra, se esta for colocada em extinção, Sniffy demonstra o bem conhecido aumento momentâneo na freqüência desta resposta em relação ao que era registrado ao final da etapa imediatamente anterior. Conforme declarado pelos autores, "com animais reais, o conceito de frustração é, às vezes, evocado como uma explicação" (itálico dos autores de Sniffy Pro) desse aumento momentâneo na ocorrência de respostas que passaram a se encontrar em extinção. Com o Sniffy, entretanto, a explicação é muito mais simples, uma vez que Sniffy Pro não inclui um fator relativo a frustração. Nesse caso, os autores são veementes em sua reivindicação de que é muito bom que o Sniffy demonstre esse aumento de respostas, uma vez que se trata de uma característica "realista". Anteriormente, os autores haviam afirmado que fizeram Sniffy propositalmente diferente; agora, orgulham-se de uma semelhança eventual e aparentemente superficial que este mantém com um rato. Esta "lógica dupla" é contraditória e preocupante. O que ela sugere é que os autores dão-se por satisfeitos com qualquer semelhança que se obtenha, independentemente do meio necessário para isso. E, assim, lá se vão os "princípios elementares sobre os quais os psicólogos concordam" (p. 3).

A possibilidade de estudo de esquemas de reforço encontra-se também disponível em Sniffy Pro. A propósito, a seção de esquemas de reforço nos preocupou bastante. Aqui, os "princípios" desapareceram definitivamente e o programa parece não fazer mais do que reproduzir registros cumulativos que, em algum nível, podem se assemelhar àqueles relativos ao comportamento de um rato. O material explicativo é superficial, embora bastante adequado (pp. 45-51) para o seu propósito, e quanto a isso não há o que se objetar. O que de fato incomoda é a simulação que se faz. Aqui, os alunos são levados a aprender erroneamente que o responder sob reforçamento intermitente depende da força da associação 
Barra-Som, isto é, a suposta magnitude momentânea do papel reforçador da comida. Em se tratando do aumento gradual do valor de um esquema de razão, por exemplo, para decidir qual o momento em que se deve aumentar o número de respostas exigidas para reforçamento, os alunos devem olhar para a Janela da Mente que suposta e ilusoriamente quantifica o grau da associação Barra-Som. Estando o grau da associação suficientemente elevado, o número de respostas para reforçamento poderia ser aumentado. Trata-se, mais uma vez, de um desvio do propósito didático, dado que o programa não aborda o motivo pelo qual essa associação adquire força no decorrer do reforçamento intermitente. Muito mais útil teria sido preocupar-se em mostrar instantaneamente que o equilíbrio que porventura exista entre extinção e reforçamento é proporcionado pelo próprio esquema. Ao atribuir um valor de força ao evento reforçador, os autores arbitrariamente estabelecem efeitos assimetricamente diferenciados da presença e da ausência de reforçamento. Essa abordagem mostra-se bastante inadequada para abordar até mesmo os fenômenos mais elementares do reforçamento intermitente.

Mais uma vez, constata-se que Sniffy Pro carece de sustentação por princípios do comportamento. O tratamento dado a esquemas de reforço, por exemplo, está baseado na concordância, isto é, na similaridade mantida pelas curvas de respostas geradas pela simulação do computador com aquelas tidas na literatura como padrões típicos de diferentes esquemas de reforço (Ferster \& Skinner, 1957, descrevem uma série de curvas que representam dados de sujeitos experimentais expostos a diferentes esquemas de reforço). Esse tratamento fica muito evidente na simulação do desempenho em esquemas de razão e intervalo fixos. Nenhum tratamento é dado à forma pela qual esses desempenhos seriam, por "princípios", produzidos. Claramente, pausas e acelerações no responder - características desses esquemas - são programadas arbitrariamente, de uma determinada maneira que os autores acreditam emular os registros cumulativos produzidos por ratos. O fato de os registros cumulativos de Sniffy Pro apresentarem variações assistemáticas é até celebrado como algo semelhante aos registros de um rato (a esse respeito, caberia retomar, novamente, as considerações que fizemos acima acerca da variação randômica do comportamento).

Em Sniffy Pro, não é apenas nos exercícios sobre esquemas de reforço e modelagem que o conhecimento em AEC é fundamentalmente concebido como uma técnica, e não como princípios e leis. Ao lidar com o condicionamento clássico, os alunos são convidados a pré-determinar, por meio da configuração de menus, a intensidade com que, após os devidos pareamentos com um estímulo incondicional, um estímulo inicialmente neutro deveria adquirir propriedades de estímulo condicional (CS) (ex., baixo, médio e alto). Entretanto, os princípios comportamentais mostram que a intensidade do CS é produto de contingências (uma variável dependente, portanto), e não uma variável pré-determinada pelo experimentador (uma variável independente). Em vez de pré-determinar resultados, saber quais contingências considerar e como estabelecê-las de modo a obter diferentes intensidades de CS seria, nesse tocante, o que de fato deveria ser a atividade didaticamente relevante para a formação dos alunos.

Por mencionar o condicionamento clássico, verificamos que os exercícios propostos em Sniffy Pro nessa esfera são, tanto quanto seja do nosso conhecimento, raramente, senão jamais, incluídos em cursos de laboratórios para estudantes em nível de graduação. Em vez de estudos do comportamento operante, a maior parte do manual trata de princípios de habituação e condicionamento clássico. Das 172 páginas com conteúdo de AEC (material explicativo, exercícios, análises), 106 tratam desses tópicos, ou 112, caso se inclua, na seção de condicionamento clássico, aquela sobre peak shift extraída do material sobre controle de estímulos operante (o que acreditamos ser uma categorização mais precisa). Em outras palavras, $62 \%$ a $65 \%$ do material trata do condicionamento clássico e não do condicionamento operante. E, contraditoriamente a esta ênfase no primeiro, parece estranho que os dados de condicionamento clássico sejam apresentados sob a forma de registros cumulativos, uma vez que pouco dessa literatura reconhece dados com esse tratamento. Caso os alunos estivessem de fato realizando os exercícios simulados por Sniffy Pro, seria pouco provável que eles viessem a observar, registrar e tratar os dados para além de cálculos de razões de supressão. Isto parece estranhamente em desacordo com o objetivo expresso pelos autores, que é o de fazer com que os alunos vivenciem aquilo que estariam de fato fazendo caso estivessem conduzindo um trabalho real de laboratório.

Ao longo dos exercícios propostos no manual do Sniffy Pro, o aluno é aconselhado a "se desligar" do Sniffy e a apenas olhar o resultado do exercício, sem a inconveniência da exibição visual das imagens do rato virtual na tela. Esse procedimento é chamado "Esconda o Sniffy". Trata-se de um artifício que simula o Sniffy se comportando longe da observação dos alunos. Essa abordagem é reveladora. A metáfora do Sniffy mostrou-se inconveniente durante todo o tempo. O melhor seria apenas observar os dados produzidos pela simulação. Será que os autores não se dão conta de que os alunos vão perceber a intenção por detrás dessa rejeição ao "sujeito experimental"? Essa estratégia de esconder o Sniffy é sugerida como uma forma de poupar tempo, o que de fato acontece. No entanto, isso somente ocorre em detrimento da qualidade da aprendizagem pois, como afirmam Boyce e Hineline, "para um analista do comportamento, aprendizagem é algo que a pessoa faz, não é uma coisa que acontece a ela" (Boyce \& Hineline, 2002, p. 215)

De acordo com os autores, "Sniffy Pro constitui um modo financeiramente viável e humano de proporcionar o acesso dos estudantes ao estudo dos condicionamentos operante e clássico que os cursos de psicologia da aprendizagem tipicamente discutem." (p. 1). Os custos financeiros de se adotar Sniffy Pro podem eventualmente ser de fato menores do que os de se manter adequadamente um laboratório de ratos. Contudo, esta contabilidade só se completa em uma análise mais ampla, na qual a efetividade das práticas de laboratório com ratos é contraposta ao comprometimento imposto por Sniffy à aprendizagem efetiva e correta do aluno. O que Sniffy Pro nos deixa, portanto, é a mensagem de que o uso de instrumentos informatizados de ensino não implica, per se, em progresso. Esta mensagem tem sido absorvida por muitos alunos que nos procuram para relatar que passaram pela experiência de utilizar o Sniffy Pro no lugar das práticas de laboratório com ratos e descrevem este instrumento como precário e ineficiente pedagogicamente. 
Finalmente, algumas pessoas argumentam que Sniffy Pro constitui uma "alternativa ética ao uso de animais na prática de ensino". Contudo, os aspectos éticos envolvidos no uso de animais na pesquisa e no ensino têm sido amplamente debatidos e regulamentados em vários países, inclusive no Brasil (veja, por exemplo, o documento "Diretrizes para a Conduta Ética no Cuidado e Uso de Animais", da American Psychological Association, APA, 2001 ${ }^{4}$, bem como as diretrizes éticas da pesquisa com animais elaboradas pelo Núcleo Interinstitucional de Biotérica do Hospital das Clínicas de Porto Alegre, HCPA, 20035). À luz das regulamentações, o uso que se faz de ratos no laboratório didático em Análise Experimental do Comportamento não só respeita absolutamente os preceitos éticos vigentes, como deveria vir acompanhado, rotineiramente, pela exposição dos alunos às questões pertinentes a este âmbito de discussão (Matos e Tomanari, 2002). Infelizmente, não é o que se contempla em Sniffy Pro. Pelo contrário, sob a perspectiva ética, e considerandose o propósito fundamentalmente didático deste aplicativo, Sniffy Pro propõe exercícios - por exemplo, aqueles que envolvem a utilização de estímulos aversivos - que seriam alvo de fortes críticas por parte de qualquer comitê de ética e, possivelmente, seriam rejeitados para fins didáticos. No entanto, sob o argumento de ser uma simulação, Sniffy Pro absorve com naturalidade tais procedimentos questionáveis e, tanto pior, exclui das atividades de laboratório a discussão ética que necessariamente deveria acompanhá-los. Exclusão como essa é inaceitável e só pode ser compreendida na medida em que o próprio uso de Sniffy Pro, por todos os seus aspectos que comprometem a aprendizagem dos alunos, seria, em si, eticamente questionável.

\section{Referências}

Alloway, T., Wilson G., Graham, J., \& Krames, L. (2000). Sniffy: The Virtual Rat - Pro Version. Belmont, CA: Wadsworth/ Thomson Learning.

APA (2001). Guidelines for ethical conduct in the care and use of animals. Obtido em janeiro de 2003 em http://www.apa.org/ science/anguide.html.

Boyce, T.E., \& Hineline, P.N. (2002). Interteaching: A strategy for enhancing the user-friendliness of behavioral arrangements in the college classroom. The Behavior Analyst, 25, 215-226.

Brown, P.L., \& Jenkins, H.M. (1968). Auto-shaping of the pigeon's key-peck. Journal of the Experimental Analysis of Behavior, $11,1-8$.

Daly, H.B., \& Daly, J.T. (1982). A mathematical model of reward and aversive nonreward: Its application in over appetitive learning situations. Journal of Experimental Psychology: General, 111, 441-480.

Ferster, C.B., \& Skinner, B.F. (1957). Schedules of reinforcement. New York: Appleton-Century-Crofts.

Gomide, P., \& Weber, L.N.D. (1998). Análise Experimental dos Comportamento: Manual de Laboratório. Curitiba: Editora da UFPR.

4. Texto elaborado por "Committee on Animal Research and Ethics (CARE)", "American Psychological Association", disponível no endereço eletrônico http://www.apa.org/science/bsaweb-care.html.

5. Texto disponível em http://www.ufrgs.br/HCPA/gppg/bioetica.htm.
Guide, M.A.A., \& Bauermeister H.B. (1979). Exercícios de laboratório em Psicologia. São Paulo: Martins Fontes.

HCPA (2003). Diretrizes éticas da pesquisa com animais. Comitê de ética do Hospital das Clínicas de Porto Alegre. Disponível em janeiro de 2003 em http://www.ufrgs.br/HCPA/gppg/ bioetica.htm.

Keller, F.S. (1987). Pedro Preguiça vai à escola. São Paulo: Edicon.

Keller, F.S. (1968). Good-bye teacher... Journal of Applied Behavior Analysis, 1, 79-89.

Kemp, S.N., \& Eckerman. D.A. (2001a). Why simulate? Revista Brasileira de Psicoterapia e Medicina Comportamental, 03, 25-35.

Kemp, S.N., \& Eckerman. D.A. (2001b). Situational descriptions of behavioral procedures: The In Situ testbed. Journal of the Experimental Analysis of Behavior, 75, 135-164.

Machado, L.M.M., \& Matos, M.A. (1990). Laboratório em cursos de graduação em Psicologia: buscando treinar atitudes. Ciência e Cultura, 42, 647-652.

Machado, A. \& Silva, F.J. (1998). Greatness and misery in the teaching of the psychology of learning. Journal of the Experimental Analysis of Behavior, 70, 215-234.

Matos, M.A. \& Tomanari, G.Y. (2002). A Análise Experimental do Comportamento no Laboratório Didático. São Paulo: Manole.

Rescorla, R.A. \& Wagner, A.R. (1972). A theory of Pavlovian conditioning: Variations in the effectiveness of reinforcement and nonreinforcement. Em A.H. Black \& W.F. Prokasy (Eds.), Classical conditioning II: Current research and theory (pp. 64-99). New York: Appleton-Century-Crofts.

Sidman, M. (1960). Tatics of scientific research. New York: Basic Books.

Skinner, B.F. (1938). The behavior of the organisms. New York: Appleton-Century-Crofts.

Thorndike, E.L. (1898). Animal intelligence: An experimental study of associative processes in animals. Psychological Monographs, 2, 8.

Tomanari, G.Y. (2000). Maximizando o uso do Laboratório Didático de Psicologia no ensino de conceitos e práticas. Em R.R. Kerbauy (org.), Sobre Comportamento e Cognição (pp. 79-83). Santo André: Set Editora. 